Saudi Journal of Oral and Dental Research

Abbreviated Key Title: Saudi J Oral Dent Res

ISSN 2518-1300 (Print) |ISSN 2518-1297 (Online)

Scholars Middle East Publishers, Dubai, United Arab Emirates

Journal homepage: https://saudijournals.com/sjodr

\title{
Association between Malocclusion and Dental Caries Experience in the Primary Dentition
} \author{
Hichem Ghedira $^{4}$ \\ ${ }^{1}$ Assistant Professor, Department of Pediatric, Faculty of Dental Medicine, Monastir, Tunisia \\ ${ }^{2}$ Resident, Department of Pediatric, Faculty of Dental Medicine, Monastir, Tunisia \\ ${ }^{3}$ Postgraduate Student, School of Dentistry, Monastir University, Monastir, Tunisia \\ ${ }^{4}$ Professor, Department of Pediatric, Faculty of Dental Medicine, Monastir, Tunisia \\ ${ }^{5}$ Professor, School of Dentistry, Monastir University, Monastir, Tunisia
}

Yamina Elelmi ${ }^{1 *}$, Raja Guetat ${ }^{2}$, Mohamed Karim Ben Salem ${ }^{3}$, Fatma Masmoudi ${ }^{4}$, Ahlem Baaziz ${ }^{4}$, Fethi Maatouk ${ }^{5}$,

DOI: $10.36348 /$ sjodr.2020.v05i01.012

| Received: 13.01.2020 | Accepted: 20.01.2020 | Published: 29.01.2020

*Corresponding author: Yamina Elelmi

\section{Abstract}

Aim: The purpose of this study was to assess the prevalence of malocclusion and to investigate its correlation with caries experience in primary dentition in 3- to 5 year-old children in the region of Monastir, Tunisia. Materials and Methods: It was a descriptive cross-sectional study, which focused on children in preschool establishment in the region of Monastir, Tunisia. It consisted on an oral examination of 393 children and a data collection through a questionnaire completed by parents. The ANOVA test and the Pearson chi-square test were used to assess the relationship between the prevalence of malocclusion and caries experience in primary dentition. Results: The prevalence of ECC was $49.9 \%$. The prevalence of malocclusion was 17, 3\%. The relationship between the prevalence of ECC and malocclusion was non-significant $(\mathrm{p}=0.578)$. An association was found between malocclusion and other factors such as breathing type and night time bottle feeding. Conclusion: The current study showed that the appearance of malocclusions was not associated with caries experience in primary dentition. Further studies are warranted to explore the risk factors of malocclusion in primary dentition.

Keywords: Malocclusion - dental caries - Primary dentition - early childhood caries - prescool children.

Copyright @ 2020: This is an open-access article distributed under the terms of the Creative Commons Attribution license which permits unrestricted use, distribution, and reproduction in any medium for non-commercial use (NonCommercial, or CC-BY-NC) provided the original author and source are credited.

\section{INTRODUCTION}

Childhood is the mirror that reflects the propensity of adulthood. An ideal primary dentition likewise is an indicator of potentially perfect permanent dentition.

This is why; recently there has been an increased awareness of the role of the primary dentition in the development and occlusion of the permanent dentition $[1,2]$.

Many epidemiological studies on prevalence of caries and malocclusion in children have been presented in the literature. However, only a few have simultaneously evaluated dental and orthodontic/ occlusal findings in an adequate sample and even fewer have analyzed possible interactions between them. It has been assumed by several authors that dental caries and premature loss of primary teeth are predisposing factors for occlusal and space anomalies in mixed and permanent dentitions [3]. However, a causal relationship between malocclusion and dental caries has not been convincingly demonstrated [4]. Previous attempts to investigate a possible association of malocclusion and dental caries have shown conflicting or inconclusive results $[5,6]$. This study aimed to assess the prevalence of malocclusion and to investigate its association with caries experience in primary dentition.

\section{MATERIALS AND METHODS}

It was a descriptive cross-sectional study that took place in preschools in the region of Monastir, Tunisia from April to June 2018.

\section{Sample Selection}

The population of this study was consisted of children aged between 3 to 5 years in temporary dentition. 
The sample size (n) was calculated according to the following equation $n=Z^{2} \times(P)(1-P) / d^{2}$, where $\mathrm{Z}=1.96$ is the confidence level for an accuracy of 0.05 , $\mathrm{p}=60.9 \%$ is the prevalence of ECC according to a similar epidemiological survey conducted in Morocco in 2013, and $\mathrm{d}=0.05$ corresponds to the chosen accuracy.

The sample size after this calculation should be at least 393 individuals.

The cluster sampling method was used for the selection of the subject.

A first draw was made to select 21 kindergartens in the various sectors of the region that can gather children from all socio-economic levels. At the level of each cluster, a second draw was made to select the eligible cluster units (small section, medium section, large section).

\section{Inclusion Criteria}

Children included in this study were:

- Any children under the age of 6 present in kindergarten at the moment of the study.

- Children with fully erupted primary dentition and without any permanent teeth.

- Children whose parents completed carefully the questionnaire.

\section{Non-Inclusion Criteria}

Children non included in this study were:

- Children with mixed dentition.

- Children who were absent during our visit.

- Children whose parents refused to participate or did not completed the questionnaire.

\section{Conduct of the study and Data Collection}

First, an approval from the regional delegation of the Ministry of Family and Child Welfare was obtained, then the heads of the kindergartens were informed about the study and consents of the parents were obtained.

During the first visit to kindergartens, questionnaires were entrusted to the teachers of these establishments to distribute them to the parents of the children, who were given an average of one week to complete them.

The questionnaire was designed focusing on sociodemographic characteristics of the children such as gender, number of siblings, monthly familial income, and parental education level. Also, the feeding methods of the children, the dental visit experience and oral hygiene measures were investigated in this questionnaire. Finally, the parents were asked regarding health characteristics of the children, sleep quality and the presence of nocturnal snoring. The second visit took place after the questionnaires were completed and collected by the teachers and consisted of two parts:

The first part was an awareness and motivation to oral hygiene with learning an appropriate brushing method. It concerned all the children present at the visit, even those whose parents did not return the questionnaire.

On the other hand, the second part concerned only children whose parents had carefully completed the questionnaire. It consisted on an oral examination conducted by a single examinator in order to identify the following clinicals data needed for the investigation:

- Dental formula and the identified conditions.

- Chapman terminal plane.

- The type of maxillary and mandibular arch.

- Breathing type.

- Presence of malocclusion / type of malocclusion.

Criteria for each type of malocclusion were defined as follows:

- Anterior cross bite: recorded when the lower incisors or canines are in front of the corresponding upper teeth.

- Edge-to-edge occlusion: recorded when the maxillary incisors and the corresponding mandibular incisors are occluded in an edgeto-edge status, without overbite or overjet.

- Open bite: recorded when the upper and lower incisors are vertically separated regardless of the extent, without any overbite. A visible space between antagonistic fully erupted canines, premolars, or molars was registered as a lateral open bite.

- Deep overbite: recorded when the vertical overlap of the incisors, when the posterior teeth are in contact is more than two thirds covered or when the overlap is more than 3 $\mathrm{mm}$, measured to the nearest $0.5 \mathrm{~mm}$.

- Posterior cross bite: recorded when the buccal cusps of the maxillary molars occlude lingually to the buccal cusps of the mandibular antagonists in at least one pair of teeth, uni or bilaterally. Teeth in an edge-to-edge position were also included [7].

\section{STATISTICAL ANALYSIS}

Questionnaire data were captured and analyzed using SPSS version 24 software and Excel 2010 software. The significance was considered for $\mathrm{p} \backslash 0.05$. The data distribution analysis was carried out using the Kolomognou-smirnov test. When the distributions were normal and the variances were equal, the results were expressed by their mean +/- the standard error of the mean. The ANOVA test and the Pearson's khi-square test were used to compare the different variables. 


\section{RESULTS}

Prevalence of ECC and dmft index

The prevalence of ECC was $49.9 \%$ and the average dmf index was $1.42+/-0.1$. In Table-1, the condition of all teeth was detailed.

Table-1: Distribution of teeth condition

\begin{tabular}{|l|l|l|}
\hline Condition of the teeth & Number & Percentage \\
\hline healthy & 7300 & $92.9 \%$ \\
\hline decayed & 515 & $6.5 \%$ \\
\hline filling & 28 & $0.4 \%$ \\
\hline missing & 17 & $0.21 \%$ \\
\hline total & 7860 & $100.0 \%$ \\
\hline
\end{tabular}

The decayed tooth component "index $\mathrm{d}$ " represented $90 \%$ of the $\mathrm{dmf}$ index since in this study 515 decayed teeth were noted for only 28 filled teeth and 17 missing teeth.

\section{Prevalence of different types of malocclusion}

Normal occlusion was observed in 325 children $(82.7 \%)$, while $68(17.3 \%)$ children had one or more types of anomalies Figure-1.

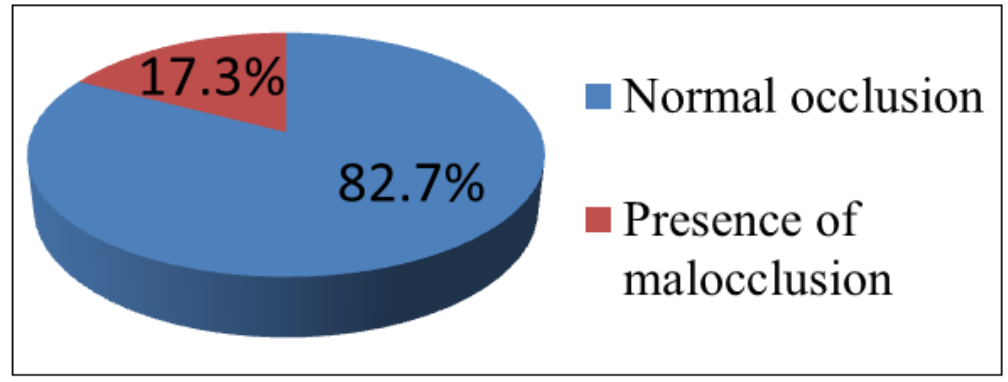

Fig-1: Prevalence of malocclusion

As shown in Table-2, the most common type of malocclusion in the sagittal direction was the edgeto-edge occlusion (22\%), while the most prevalent malocclusions in the vertical and transverse directions were deep overbite $(17.7 \%)$ and posterior cross bite $(11.8 \%)$. The prevalence of anterior crossbite, anterior open bite, lateral open bite and general cross bite was $11.8,14.7,4.4$ and $8.8 \%$, respectively. The prevalence of the maxillary exognathia was the lowest $(1.5 \%)$.

Table-2: Prevalence of different types of malocclusion

\begin{tabular}{|l|l|l|}
\hline Type of malocclusion & $\mathbf{N}$ & Percentage (\%) \\
\hline Not specified & 5 & $7,3 \%$ \\
\hline Anterior open bite & 10 & $14,7 \%$ \\
\hline Lateral open bite & 3 & $4,4 \%$ \\
\hline Edge to edge occlusion & 15 & $22 \%$ \\
\hline Maxillary exognathia & 1 & $1,5 \%$ \\
\hline Anterior cross bite & 8 & $11,8 \%$ \\
\hline General cross bite & 6 & $8,8 \%$ \\
\hline Posterior cross bite & 8 & $11,8 \%$ \\
\hline Deep overbite & 12 & $17,7 \%$ \\
\hline Total & 68 & $100 \%$ \\
\hline
\end{tabular}

Relationship between Presence of Malocclusion and ECC

The statistical relationships between the prevalence of ECC and the presence of malocclusion, as well as that between the average dmf index and the presence of malocclusion were non-significant ( $\mathrm{p}=0.578$ and $\mathrm{p}=0.89$ respectively) Table- 3 .
Table-3: Prevalence of different types of malocclusion

\begin{tabular}{|l|ll|l|l|}
\hline \multicolumn{4}{|l|}{ MALOCCLUSION } \\
\hline Variable & & Ddl & VALUE & $\mathrm{P}^{*}$ \\
\hline ECC prevalence & $\mathbf{1}$ & & $\mathbf{0 , 3 1 0}$ & $\mathbf{0 , 5 7 8}$ \\
\hline Mean dmf & $\mathbf{1}$ & $\mathbf{0 , 0 2}$ & $\mathbf{0 , 8 9}$ \\
\hline
\end{tabular}

Relationship between different types of malocclusion and other factors

As shown in Table-4, the presence of malocclusions was more important for children with an asymmetric Chapman terminal plane $(38,5 \%)$ than those with a mesial step $(15.7 \%)$ or flush terminal plane $(19.4 \%)$. The relationship between malocclusion and the type of Chapman terminal plane was not statistically significant $(\mathrm{p}=0.1)$.

A statistically significant relationship was found between malocclusion and breathing type $(p=0.045)$. In fact, $33.3 \%$ of oral respirators had malocclusion, while the percentage of malocclusion for mixed respirators and for children with nasal respiration was respectively $15.8 \%$ and $16.9 \%$.

An association was found between malocclusion and nighttime bottle-feeding $(\mathrm{p}=0.036)$. In fact, children who used to take the bottle before sleeping had almost twice as many malocclusions with a value of $21.1 \%$ while children who did not use it had only $13 \%$ malocclusions. 
Yamina Elelmi et al; Saudi J Oral Dent Res, Jan 2020; 5(1): 71-75

Table-4: Association between independent variables and malocclusion in children aged between 3 to 5 years

\begin{tabular}{|c|c|c|c|}
\hline \multicolumn{2}{|l|}{ Variables } & Malocclusion & (p) \\
\hline \multirow[t]{3}{*}{ Chapman terminal plane } & Mesial step & $15.7 \%$ & \multirow[t]{3}{*}{0.1} \\
\hline & Distal step & $19.4 \%$ & \\
\hline & Asymétric & $38.5 \%$ & \\
\hline \multirow[t]{3}{*}{ Breathing type } & Nasal & $16.9 \%$ & \multirow[t]{3}{*}{ 0.045* } \\
\hline & Mixed & $15.8 \%$ & \\
\hline & Buccal & $33.3 \%$ & \\
\hline \multirow[t]{2}{*}{ Breastfeeding } & Yes & $16.7 \%$ & \multirow[t]{2}{*}{0.31} \\
\hline & No & $24.1 \%$ & \\
\hline \multirow[t]{2}{*}{ Night time bottle feeding } & Yes & $21.1 \%$ & \multirow[t]{2}{*}{$\underline{0.036 *}$} \\
\hline & No & $13.1 \%$ & \\
\hline
\end{tabular}

\section{DISCUSSION}

In the current study, $52.9 \%$ of the subjects who had malocclusion had dental caries. But, the relationship between dental caries and malocclusion was statistically insignificant $(\mathrm{p}=0.578)$.

A study conducted by Stahl et al., [5] in the city of Rostock, Germany did not show a statistically significant association between caries and clinical orthodontic abnormalities in temporary dentition. But, statistically significant parallelism in prevalence of malocclusion and caries were found in children with mixed dentitions. In fact, Stahl and Grabowski reported that dental caries and premature loss of primary teeth are predisposing factors for occlusal and space anomalies in the mixed and permanent dentition. In addition, Meer $\mathrm{Z}$ et al., [8] explained the high prevalence of crowding in permanent dentition by the cascading effect of caries in deciduous molars followed by extraction, and the migration of the first permanent molars, resulting in inclination and rotation of teeth in the anterior sextant. So, the long-term effects of decayed dentition influences become evident not in temporary dentition, but at later developmental stages [5].

This observation differs from the findings of Zhou et al., [9] who found that caries experience in the primary dentition was associated with malocclusion.

The overall prevalence of malocclusion $(17.3 \%)$ registered among children in the current study was much inferior than that in the Brazilian population $(46.2 \%)$ and French population (57.6\%). Higher incidence of malocclusion was reported in Chinese children $(66.31 \%)$ [10, 9, 11]. The differences between this study and other published articles could be attributed to differences in sample size, variations in ethnic group, registration method, or it could be due to the use of variable criteria for the classification of malocclusion.

Further statistical analysis revealed that other factors such as type of breathing and nighttime bottlefeeding in the primary teeth were associated with malocclusion among these children.
The current study showed an association between the presence of malocclusions and the type of breathing $(\mathrm{p}=0.045)$. These findings were in agreement with the results of Zhou et al., [9]. This association could be explained by the fact that nasal or mixed respiration contributes to the growth of the maxilla and thus allows to obtain the necessary space for a correct positioning of the teeth.

An association was found between the presence of malocclusion and nighttime bottle-feeding which could be explained by the prolonged interposition of the teat of the bottle between the two arcades, which ends by distorting the teeth and create anterior open bite.

\section{CONCLUSION}

The present data showed that caries experience has not significant influence on formation of malocclusion in primary dentition. However, other significant associations merit further studies regarding other factors that can influence dentition development. The relationship between caries experience and malocclusion should be assessed in a wider context of socioeconomic status and background factors.

\section{REFERENCES}

1. Sriram, C. H., Priya, V. K., Sivakumar, N., Reddy, K. M., Babu, P. J., \& Reddy, P. (2012). Occlusion of primary dentition in preschool children of Chennai and Hyderabad: A comparative study. Contemporary clinical dentistry, 3(1), 3137.

2. Wagner, Y., \& Heinrich-Weltzien, R. (2015). Occlusal characteristics in 3-year-old childrenresults of a birth cohort study. BMC Oral Health, 15(1), 94.

3. Masmoudi, F., Elelmi, Y., Ben Amara, S., Chouchene, F., \& Maatouk, F. (2017). Premature loss of temporary mandibular molars: study of the effects on the arch. Tunisian Dental Journal, 7(1):14-20.

4. McLain, J. B., \& Proffit, W. R. (1985). Oral health status in the United States: prevalence of malocclusion. Journal Dent Educ, 49(6):386-396. 
5. Stahl, F., \& Grabowski, R. (2004). Malocclusion and caries prevalence: is there a connection in the primary and mixed dentitions?. Clinical oral investigations, 8(2), 86-90.

6. Helm, S., \& Petersen, P. E. (1989). Causal relation between malocclusion and caries. Acta Odontologica Scandinavica, 47(4), 217-221.

7. Björk, A., Krebs, A. A., \& Solow, B. (1964). A Method for Epidemiological Registration of Malocculusion. Acta Scandinavica, 22(1), 27-41.

8. Meer, Z., Sadatullah, S., Wahab, M. A., Mustafa, A. B., Odusanya, S. A., \& Razak, P. A. (2016). Prevalence of malocclusion and its common traits in Saudi males of Aseer region. Journal of Dental Research and Review, 3(3), 99-102.

9. Zhou, Z., Liu, F., Shen, S., Shang, L., Shang, L., \& Wang, X. (2016). Prevalence of and factors affecting malocclusion in primary dentition among children in Xi'an, China. BMC Oral Health, 16(1), 91.

10. Carvalho, A. C., Paiva, S. M., Scarpelli, A. C., Viegas, C. M., Ferreira, F. M., \& Pordeus, I. A. (2011). Prevalence of malocclusion in primary dentition in a population-based sample of Brazilian preschool children. European journal of paediatric dentistry: official journal of European Academy of Paediatric Dentistry, 12(2), 107-111.

11. Thilander, B., Pena, L., Infante, C., Parada, S. S., \& de Mayorga, C. (2001). Prevalence of malocclusion and orthodontic treatment need in children and adolescents in Bogota, Colombia. An epidemiological study related to different stages of dental development. European journal of orthodontics, 23(2), 153-168. 